\title{
SUCCESSFUL OPERATION OF THE 500 MHZ SRF MODULE AT TLS
}

\author{
Ch. Wang", L.H. Chang, S.S. Chang, F.T. Chung, F.Z. Hsiao, G.Y. Hsiung, K.T. Hsu, C.C. Kuo, H.C. \\ Li, M.C. Lin, R.J. Lin, Y.K. Lin, G.H. Luo, M.H. Tsai, J.Y. Yang, T.T.Yang, M.S Yeh and C.T. Chen, \\ National Synchrotron Radiation Research Centre, Hsinchu 30076, Taiwan
}

\begin{abstract}
A project to replace two existing room-temperature radio frequency (RF) cavities by one CESR-III $500 \mathrm{MHz}$ superconducting radio frequency (SRF) module was initiated for the Taiwan Light Source (TLS) synchrotron ring in 1999. The goals are to double the photon flux of the synchrotron light by doubling the electron beam current and to increase the stability of the electron beam by taking the advantage of the ultra-weak high-order modes (HOM) of the SRF cavity. The SRF module has been routinely operated since February 2005. The NSRRC users have benefited from a very high photon flux stability $\left(\Delta \mathrm{I}_{0} / \mathrm{I}_{0} \sim 0.05 \%\right)$ that had never been achieved previously. Here, we report the initial operational experience of the SRF system.
\end{abstract}

\section{SRF PROJECT AT NSRRC}

Since the successful commissioning of the TLS storage ring in 1993, the electron beam has suffered from strong coupled-bunch instabilities caused by the two existing room-temperature RF cavities designed in the 1970s. Many attempts, including the improvement of the cavity cooling system, addition of a higher order mode (HOM) tuner, and the application of RF modulation technique, have been made to improve significantly the electron beam stability. Various efforts to increase the photon flux and brightness have also been carried out, but only a limited improvement was achieved. In 1999, a major accelerator upgrade project [1] was initiated with the goals to double the TLS photon flux by increasing the electron beam current up to $500 \mathrm{~mA}$ and to improve

Table 1: Parameters that govern the operation of the CESR-III 500MHz srf module at TLS.

\begin{tabular}{|l|l|}
\hline Nominal Machine Energy & $1.5 \mathrm{GeV}$ \\
\hline Revolution Frequency & $2.49827 \mathrm{MHz}$ \\
\hline Maximum Beam Current & $<500 \mathrm{~mA}$ \\
\hline SR Energy Loss per Turn & $<164 \mathrm{keV}$ \\
\hline RF Harmonic Number & 200 \\
\hline Beam Power & $<82 \mathrm{~kW}$ \\
\hline RF Frequency & $499.654 \mathrm{MHz}$ \\
\hline RF Voltage & $1.6 \mathrm{MV}$ \\
\hline Number of RF Cavities & 1 \\
\hline R/Q per Cell $\left(\mathrm{V}^{2} / 2 \mathrm{Pc}\right)$ & $89 / 2$ \\
\hline Nominal External Q & $2.5 \mathrm{E} 5$ \\
\hline Cryogenic Static Load & $<30 \mathrm{~W} @ 4.5 \mathrm{~K}$ \\
\hline
\end{tabular}

${ }^{\#}$ rfwang@nsrrc.org.tw

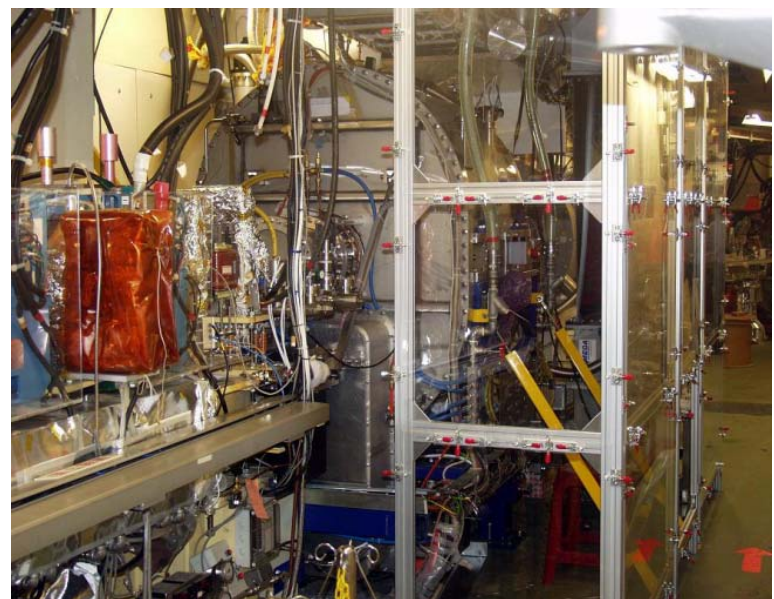

Figure 1: SRF module in routine operation at NSRRC.

substantially the electron beam stability by eliminating the coupled bunch instabilities caused mostly by the HOMs of the room-temperature RF cavities.

After the SRF module was successfully installed and commissioned in November-December of 2004, the NSRRC entered its new era of SRF operation. During the 2005 January-February scheduled shutdown for installing the new kickers for top-up injection operation, the SRF module was warmed up to $300 \mathrm{~K}$ to release the residual gases that were condensed onto its cryogenic inner surface during the commissioning. By using the SRF module and an existing $60 \mathrm{~kW}$ RF transmitter, the TLS has reached $240 \mathrm{~mA}$ beam current in top-up mode and $280 \mathrm{~mA}$ in decay mode operation. The machine is now operated routinely with a beam current of $200 \mathrm{~mA}$ in decay mode. Table I gives the operation parameters.

A maximum beam current of $500 \mathrm{~mA}$ will be achievable at the TLS, when the new $100 \mathrm{~kW}$ klystron manufactured by CPI is conditioned and attached to the SRF module. The goal is to achieve a routine operation of $350 \mathrm{~mA}$ beam current in top-up mode before the end of 2005. Factors such as integrated radiation dose, the temperature of the electron beam vacuum chambers, and the capacity of the active feedback system to stabilize the residual beam instabilities may slow down the progress toward reaching the targeted beam current. Further improving the injection efficiency and increasing the beam lifetime is crucial to achieve the project goal of operating TLS at $500 \mathrm{~mA}$ beam current in top-up injection mode. Figure 1 shows the SRF module in operation at TLS.

The major achievements of the SRF project in the last five years are summarized as following. 
The two existing room-temperature cavities were replaced by one most-recently-developed SRF module, as mentioned above. The fabrication of the SRF modules was contracted out to ACCEL after the NSRRC received a technical transfer from Cornell University. A number of difficulties were encountered during the production. Major faults are the buckling of the niobium waveguide during warm high-pressure cryogenic safety test and the cracking of the RF ceramic window during the high power RF processing. The delivery of the second SRF module is pending because of an unexpected quenching at extremely low field gradient owing to indium contamination.

A cryogenic plant has been built and installed by AirLiquide to support the operation of the SRF, according to the NSRRC's specifications and configurations, as displayed in Figure 2. The final performance fulfils most of our design goals and the requirements of SRF operation at synchrotron light sources. These include (a) the capability of long-term continuous operation by using a turbine cold box instead of a piston one, (b) isolating vibration from the helium compressor by locating it far away from the storage ring, (c) minimizing the two-phase mixture of the supply liquid helium and the drop in helium return pressure by locating the cold box close to and higher than the SRF module, (d) conveniences of operation by exploiting fully automatic control, (e) high redundancy of helium cooling capacity by a safety factor of 1.5 and high redundancy of helium gas inventory capable of sustaining the loss of all of the helium in the SRF module once, (f) fast cooling with the help of a 2000 $\mathrm{L}$ main dewar, and (g) cryogenic load matching with frequency driver in various operational modes to save energy. Additional efforts are required to solve other problems such as minimizing the huge consumption rate of LN2 through the long transfer line, stabilizing the LN2 supply pressure into the SRF module by installing a phase separator near the SRF module, preventing a helium pressure surge caused by poor coordination between the main compressor and the helium gas manager during shutdown/failure, using an effective motor bearing in the main helium compressor that is reliable when the cryogenic plant is operated at full-power.

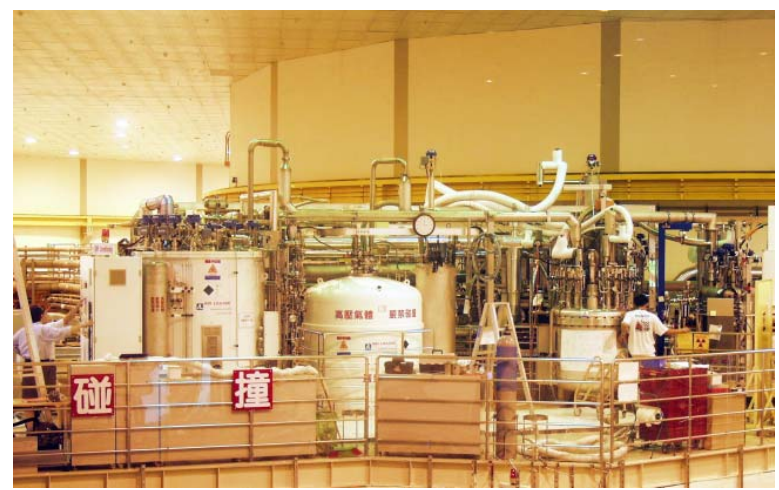

A test run of the cryogenic plant and cryogenic valve box was conducted using a $500 \mathrm{~L}$ test dewar with a builtin heater to simulate the SRF operation before the actual SRF module was attached to the cryogenic plant. Associated cryogenic electronics and PID controls were developed in-house.

A new RF plant was developed in-house and tested thoroughly for SRF operation concurrently with the production of SRF modules. A new $100 \mathrm{~kW}$ crowbar type RF transmitter was assembled in-house, with emphasis on the reduction of RF noise and operational reliability. Thanks to this independent RF plant, the system integration and the high-power test run of the SRF module were completely independent on the synchrotron routine operation. Most individual interface problems were solved during the test run without interrupting the synchrotron operation, significantly reducing the time required for commissioning the SRF module while installed in TLS.

A training program on SRF technology from module production to operation has been conducted with strong supports from Cornell. Presently, the built-up of SRF technology is focussing on the re-assembly of a used SRF module purchased from Cornell.

Intensive 3D numerical simulations including verifications of magnetic shielding using TOSCA, the Qext calculations of the CESR-III SRF module using HFSS, the bucking simulations of $\mathrm{Nb}$ waveguide using ANSYS and impedance calculations of the completed module using Gdfidl were carried out during the execution of the project.

System integration and commissioning of the SRF module, the RF plant, and the cryogenic plant were completed by NSRRC. A convenient diagnostic system that incorporates various data acquisition systems with different sampling rates has also been developed in-house to facilitate the analysis of SRF trip events.

\section{FIRST OPERATIONAL EXPERIENCE}

Synchrotron light users around the world demand continuously higher and higher photon flux intensity and stability. Although the use of SRF technology in synchrotron light sources can improve immensely both the photon flux intensity and stability, it also comes with crucial challenges on machine reliability and availability. Our experience of operating the TLS with a SRF module in the last three months and the intense effort to improve its reliability are described below.

The trip rate (or mean time between failure) and mean time to repair are measures for the machine reliability and availability. After 12 years of ongoing efforts to improve the existing room-temperature RF system at TLS, its reliability has become excellent. For instance the mean

Figure 2: Cryogenic plant designed for srf module. 


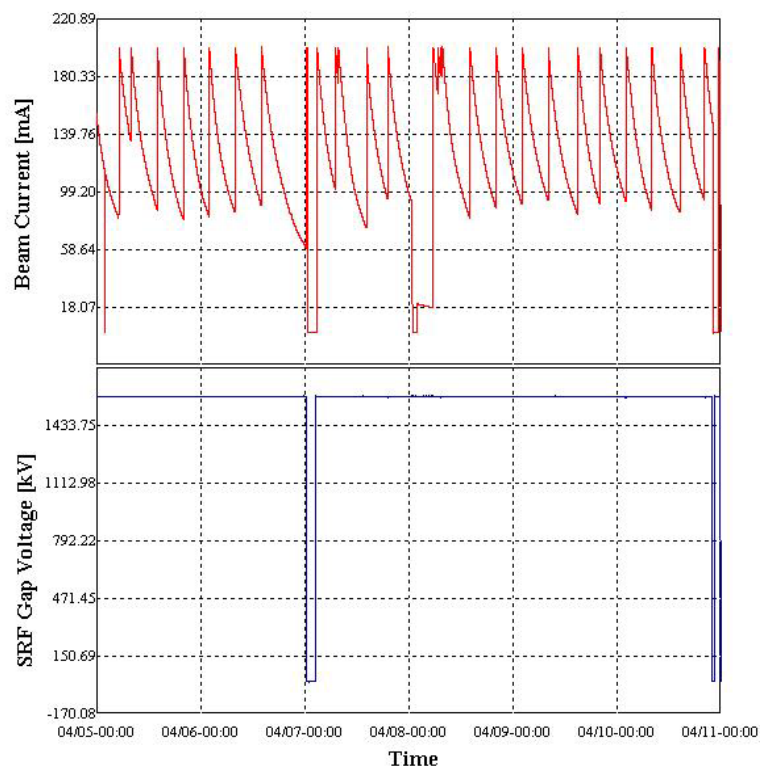

Figure 3: Summary of machine operations in a typical week.

trip rate of the two complete room-temperature RF plants (cavities plus transmitters) in 2004 was around 1.5 trip/month. With SRF operation, the mean trip rate of a complete SRF plant is more than once per week during the user shifts. This value is eight times higher than that in 2003 when the two room-temperature cavities were in operation. Figure 3 displays a summary of the machine operations in a typical week (Monday is often scheduled for machine study or maintenance).

Of all the trip events in the user shifts, two thirds were related to the SRF module and the rest were associated with other parts of the RF plant. SRF trips fall into two major categories: quenching and window arcing. Most non-SRF trips are caused by arcing of the waveguide circulator and klystron's modulator (accelerator) current high. We speculate that both the window arcing (three sensors) and circulator arcing are due to false alarms because the immunity of the fast TTL electronic circuit boards to electro-magnetic interference (EMI) is poor. Note that the maximum RF operating power is low (under $50 \mathrm{~kW}$ ) for the high-power RF components capable for $200 \mathrm{~kW}$. Window arcing or waveguide circulator arcing (all in the AFT design) also occurs when the RF power is off. Two of the three window arc detectors have never fired arcing signal simultaneously after the cabling routes have been improved and ferrite beads inserted into the circuit AC power lines. Two consequent arcing at same locations were never observed within $1 \mathrm{~ms}$. Meanwhile, no effective solution has been found yet to solve the problem of false alarms from window or circulator arcing. Dividing the fibre optic signal into two independent arc detectors may provide a plausible verification of current false alarm. The reason for the high klystron modulator (accelerator) current is unknown. The operating klystron was contaminated in the past and repaired following a few months of HV conditioning in-house. The problem may be of the klystron itself or of a false alarm.

SRF quenching is a real event in which a quench detector determining a "quenching" when the measured accelerating voltage of the SRF cavity drops by more than $30 \%$ from its set value. Falls in the cavity voltage are not caused by the quenching of superconductivity, but by the fluctuations of the cavity voltage, when either the cavity is detuned abruptly or the circulating electron beam is extremely unstable (such as in case of beam loss). Microphonics is a likely cause of fast detuning of the cavity resonance, which cannot be compensated for by the cavity mechanical tuner loop of the low-level RF system. A CESR-III piezo tuner will be installed for diagnostic purposes. Fluctuations of the supply pressure of the LN2 to the SRF module or the acoustic thermal oscillations that develop in the LN2 cooling channels around the waveguide double-elbow are the most likely sources of microphonics. An upgrade of the existing low-level RF system based on a 1980s' design is being studied. Currently, such "quenching" is minimized by reducing the tuner loop gain.

\section{CONCLUSION}

In 1999, a bold decision was made at NSRRC to adopt SRF technology in a major TLS machine upgrade. Nowadays, the SRF cavity has become the preferred choice in building or planning new light sources worldwide. As pioneers in using a SRF module as an accelerating cavity for a third-generation light source, we are pleased to report that the users are satisfactory with the SRF performance. Mastering the SRF technology inhouse is vital to the realization of a highly reliable and optimal operation of the SRF module in an accelerator. Bridging the SRF technical gap quickly remains a major challenge for our technical staff and requires ongoing intense effort.

\section{ACKNOWLEDGMENTS}

We are grateful to $\mathrm{H}$. Padamsee for his continuous support and encouragement on our SRF project. The continued technical support of S. Belomestnykh, J. Sears, and P. Quigley (Cornell Univ.) is especially appreciated. We sincerely thank T. Furuya (KEKB), R. Lange (DESY), J. Mulholland (ACT), and K. C. Wu (BNL) for valuable technical consultations, and to M. Pekeler (ACCEL) and A. Caillaud (AirLiquide) for many valuable discussions and constructive comments.

\section{REFERENCES}

[1] Ch. Wang et al., "Superconducting RF Project at the Synchrotron Radiation Research Center," 10th SRF Workshop, Tsukuba, Sept. 2001, p. 34. 